\title{
Institution Quality and Social Integration of Migrant Workers
}

\author{
Xiaolian Liu ${ }^{1}$, Xiaobing Huang ${ }^{2,}$ a, *
}

\author{
${ }^{1}$ School of History and Tourism, Gannan Normal University, Ganzhou 341000, China; \\ ${ }^{2}$ School of Business, Gannan Normal University, Ganzhou 341000, China. \\ *, a Huang Xiaobing@163.com
}

Keywords. Institution Quality; Group Heterogeneity; Migrant Workers; Social Integration

\begin{abstract}
This paper comparatively analyzes the situation and impacting factors of social integration of migrant workers from group heterogeneity perspective. The study indicates that the institution quality can promote social integration of migrant workers significantly, income, institution quality and social network are the three most important factors. Household Registration System play the most important role among all these institutions affecting social integration, social insurance ranks second, however, the importance of education is decreasing gradually.
\end{abstract}

\section{Introduction}

Since the reform and opening up, a large number of rural labor forces leave the rural areas to work in the city, forming a special social group, known as the "migrant workers". According to the statistics released by the national bureau of statistics, the total number of migrant workers in China totaled 2.817 million in 2016, an increase of 4.24 million over the previous year, an increase of 1.5 percent. The realization of integration into the city is the major issue facing China's urbanization process. The integration degree is affected by the institutional factors, especially in China, there are some institutional obstacles preventing the migrant workers to be citizens, such as the Hukou system (Myers et al., 2009). In this background, this paper studies the impact of institutional quality on the social integration of migrant workers in China using a survey data.

\section{Measure}

We utilize a five-response questionnaire conforming to the Likert scale with reference to the literature on measures of social integration. The survey was conducted by the School of Business of Gannan Normal University from May to August 2013 in the Jiangxi province, Guangdong province, Henan province and Helongjiang province with face-to-face interviews. We finally acquire survey data with 869 effective samples after excluding 131 ineligible correspondents. We propose an indicator consisting of five components to measure the social integration of Chinese migrant workers including social distance, cultural integration, psychological integration, social interaction and settlement (Nesdale et al., 2000; Zagefka et al., 2002).

We employ an exploratory factor analysis method using principal-component analysis and Varimax with Kaiser Normalization. The "eigenvalue greater than 1" criterion is adopted to determine the number of factors to extract. The factor analysis is implemented in SPSS, Version 18.0. SPSS reports a Kaiser-Meyer-Olkin (KMO) index of 0.726 and an associated Barlett chi-square test which is significant at the $1 \%$ level $(\mathrm{P}<0.001)$, thereby indicating that the correlation matrix was factorable. The scree test yields a solution for all five factors with eigenvalue greater than 1 . This five-factor solution accounts for $62.458 \%$ of the variance.

The factor analysis in Table 1 shows that the four items we designate to measure the social distance have high loadings ranging from 0.709 to 0.814 , indicating that these four items can explain and define social integration well. Analogously, we observe that the items we choose for other aspects of social integration enjoy high loadings. As a consequence, we could perfectly combine all of the 16 
items into 5 common factors and use them to measure the social integration of Chinese migrant workers.

Table 1. The factor analysis

\begin{tabular}{|c|c|c|c|c|c|c|}
\hline & Variable & $\begin{array}{c}\mathrm{F} 1 \\
(2.29895) \\
\end{array}$ & $\begin{array}{c}\mathrm{F} 2 \\
(1.55486)\end{array}$ & $\begin{array}{c}\mathrm{F} 3 \\
(1.44442) \\
\end{array}$ & $\begin{array}{c}\mathrm{F} 4 \\
(1.22029) \\
\end{array}$ & $\begin{array}{c}\mathrm{F} 5 \\
(1.03679) \\
\end{array}$ \\
\hline \multirow{4}{*}{$\begin{array}{c}\text { Social } \\
\text { distance }\end{array}$} & Local residents discriminate against us & .814 & -.068 & .042 & -.053 & -.059 \\
\hline & I don't belong in the city & .805 & .070 & .014 & -.042 & -.015 \\
\hline & I feel like a stranger & .742 & -.062 & .016 & -.108 & -.008 \\
\hline & It is difficult to get along with residents & .709 & .016 & .013 & .024 & -.029 \\
\hline \multirow{3}{*}{$\begin{array}{l}\text { Cultural } \\
\text { integration }\end{array}$} & $\begin{array}{l}\text { It is important to behave according to } \\
\text { hometown norms }\end{array}$ & -.041 & .866 & .052 & .155 & .124 \\
\hline & $\begin{array}{l}\text { It is important to keep the hometown } \\
\text { lifestyle }\end{array}$ & .083 & .819 & .169 & .031 & .074 \\
\hline & It is important to obey hometown customs & -.081 & .789 & .031 & .106 & .137 \\
\hline \multirow{4}{*}{$\begin{array}{l}\text { Psychological } \\
\text { integration }\end{array}$} & $\begin{array}{c}\text { Your children should speak the hometown } \\
\text { dialect }\end{array}$ & .021 & .092 & .811 & -.064 & .033 \\
\hline & I feel like I am a member of the local city & .088 & .057 & .752 & .110 & -.003 \\
\hline & I consider myself a city dweller & -.072 & .014 & .740 & -.076 & .110 \\
\hline & I think I belong to the local city & .057 & .103 & .549 & .292 & .034 \\
\hline \multirow{3}{*}{ Social interaction } & I can speak the local dialect & -.107 & .092 & .004 & .775 & .128 \\
\hline & I am familiar with local customs & .043 & .128 & .122 & .719 & -.008 \\
\hline & I have frequent contact with city dwellers & -.152 & .039 & .001 & .561 & .331 \\
\hline \multirow[b]{2}{*}{ Settlement } & I want buy an apartment in the city & -.046 & .109 & .079 & .160 & .859 \\
\hline & $\begin{array}{c}\text { I want to settle down and develop a career } \\
\text { in the city }\end{array}$ & -.025 & .212 & .083 & .127 & .838 \\
\hline \multicolumn{2}{|r|}{ Proportion } & 20.641 & 15.733 & 10.871 & 8.571 & 6.641 \\
\hline \multicolumn{2}{|r|}{ Cumulative } & 20.641 & 36.374 & 47.245 & 55.817 & 62.458 \\
\hline \multicolumn{2}{|r|}{ Cronbach $\alpha$} & .774 & .704 & .799 & .565 & .736 \\
\hline
\end{tabular}

\section{Results}

In order to analyze the influence of various factors on the social integration of migrant workers, this paper sets the following equation:

$$
\begin{aligned}
S I_{i}= & \beta_{0}+\beta_{1} \text { age }_{i}+\beta_{2} \text { gen }_{i}+\beta_{3} \text { edu }_{i}+\beta_{4} \text { tim }_{i}+\beta_{5} \text { inc }_{i}+\beta_{6} \text { con }_{i} \\
& +\beta_{7} \text { lei }_{i}+\beta_{8} \text { mar }_{i}+\beta_{9} \text { ins }_{i}+\beta_{10} \text { sr }_{i}+\varepsilon_{i}
\end{aligned}
$$

Where dependent variable $\mathrm{SI}_{\mathrm{i}}$ denotes the social integration of migrant workers. ins ${ }_{i}$ is the variable of institutional quality, which is measured by the scores of relevant questions.

Table 2 shows the estimated results using robust OLS estimation to control the heteroscedasticity.. 
Table 2. Basic regression results

\begin{tabular}{|c|c|c|c|c|c|c|}
\hline & $\begin{array}{c}1 \\
\text { Social } \\
\text { integration } \\
\end{array}$ & $\begin{array}{c}2 \\
\text { Social } \\
\text { distance } \\
\end{array}$ & $\begin{array}{c} \\
\text { Cultural } \\
\text { integration } \\
\end{array}$ & $\begin{array}{c}4 \\
\text { Psychological } \\
\text { integration } \\
\end{array}$ & $\begin{array}{c}5 \\
\text { Social } \\
\text { interaction } \\
\end{array}$ & $\begin{array}{c}6 \\
\text { Settlement }\end{array}$ \\
\hline age & $\begin{array}{c}-0.048 * * * \\
(-0.017)\end{array}$ & $\begin{array}{l}-0.071^{*} \\
(0.036)\end{array}$ & $\begin{array}{c}-0.132 * * * \\
(0.027)\end{array}$ & $\begin{array}{l}-0.051^{*} \\
(-0.034)\end{array}$ & $\begin{array}{c}-0.011 \\
(-0.022)\end{array}$ & $\begin{array}{l}0.02^{*} \\
(0.034)\end{array}$ \\
\hline $\operatorname{sex}$ & $\begin{array}{l}0.063 * \\
(0.034)\end{array}$ & $\begin{array}{c}0.353 * * * \\
(0.072)\end{array}$ & $\begin{array}{c}0.018 \\
(0.055)\end{array}$ & $\begin{array}{l}-0.036^{*} \\
(-0.069)\end{array}$ & $\begin{array}{c}0.13 * * * \\
(0.045)\end{array}$ & $\begin{array}{c}-0.159 \\
(-0.069)\end{array}$ \\
\hline education & $\begin{array}{c}0.09 * \\
(0.018)\end{array}$ & $\begin{array}{l}0.049^{*} \\
(0.039)\end{array}$ & $\begin{array}{l}0.005^{*} \\
(0.029)\end{array}$ & $\begin{array}{c}0.075^{* *} \\
(0.037)\end{array}$ & $\begin{array}{l}0.043^{*} \\
(0.024)\end{array}$ & $\begin{array}{l}0.057 * \\
(0.037)\end{array}$ \\
\hline duration & $\begin{array}{c}0.003 \\
(0.015)\end{array}$ & $\begin{array}{c}-0.08 \\
(0.031)\end{array}$ & $\begin{array}{c}-0.054 \\
(-0.024)\end{array}$ & $\begin{array}{l}0.082 \\
(0.03)\end{array}$ & $\begin{array}{c}0.011 \\
(0.019)\end{array}$ & $\begin{array}{l}0.058^{*} \\
(0.03)\end{array}$ \\
\hline martial & $\begin{array}{c}0.086^{* *} \\
(0.042)\end{array}$ & $\begin{array}{c}0.036 * * \\
(0.088)\end{array}$ & $\begin{array}{c}0.175^{* * * *} \\
(0.066)\end{array}$ & $\begin{array}{l}0.21 * * \\
(0.084)\end{array}$ & $\begin{array}{c}0.014 * * \\
(0.054)\end{array}$ & $\begin{array}{l}0.071 * * \\
(0.084)\end{array}$ \\
\hline income & $\begin{array}{l}0.26^{* * * *} \\
(0.017)\end{array}$ & $\begin{array}{l}0.144 * \\
(0.037)\end{array}$ & $\begin{array}{c}0.02 * \\
(0.028)\end{array}$ & $\begin{array}{l}0.033^{*} \\
(0.035)\end{array}$ & $\begin{array}{l}0.035^{*} \\
(0.023)\end{array}$ & $\begin{array}{c}0.11^{*} \\
(0.035)\end{array}$ \\
\hline consumption & $\begin{array}{l}0.024 * * \\
(0.012)\end{array}$ & $\begin{array}{l}0.026^{*} \\
(0.026)\end{array}$ & $\begin{array}{l}0.016^{*} \\
(0.02)\end{array}$ & $\begin{array}{l}0.045^{*} \\
(0.025)\end{array}$ & $\begin{array}{c}0.054 * * * \\
(0.016)\end{array}$ & $\begin{array}{l}-0.018 \\
(-0.025)\end{array}$ \\
\hline leisure & $\begin{array}{l}0.08 * * \\
(0.031)\end{array}$ & $\begin{array}{c}0.117 * * \\
(0.066)\end{array}$ & $\begin{array}{l}0.042 \\
(0.05)\end{array}$ & $\begin{array}{c}0.023 \\
(0.064)\end{array}$ & $\begin{array}{c}0.100 * * \\
(0.041)\end{array}$ & $\begin{array}{c}0.121 \\
(0.063)\end{array}$ \\
\hline institution & $\begin{array}{c}0.175^{* * * *} \\
(0.022)\end{array}$ & $\begin{array}{l}0.154^{*} \\
(0.047)\end{array}$ & $\begin{array}{c}0.11^{* * * *} \\
(0.036)\end{array}$ & $\begin{array}{l}0.069^{*} \\
(0.045)\end{array}$ & $\begin{array}{c}0.068 * * \\
(0.029)\end{array}$ & $\begin{array}{l}0.072^{*} \\
(0.045)\end{array}$ \\
\hline interaction & $\begin{array}{c}0.145^{* * * *} \\
(0.014) \\
\end{array}$ & $\begin{array}{c}0.082 * * * \\
(0.029) \\
\end{array}$ & $\begin{array}{l}0.049 * * \\
(0.022) \\
\end{array}$ & $\begin{array}{c}0.165 * * * \\
(0.028) \\
\end{array}$ & & $\begin{array}{c}0.183 * * * \\
(0.028) \\
\end{array}$ \\
\hline $\begin{array}{l}\text { Observations } \\
\text { R-squared }\end{array}$ & $\begin{array}{c}798 \\
0.555 \\
\end{array}$ & $\begin{array}{c}801 \\
0.574 \\
\end{array}$ & $\begin{array}{c}799 \\
0.552 \\
\end{array}$ & $\begin{array}{c}801 \\
0.588 \\
\end{array}$ & $\begin{array}{c}801 \\
0.403 \\
\end{array}$ & $\begin{array}{c}800 \\
0.487 \\
\end{array}$ \\
\hline
\end{tabular}

Note: standard errors are included in the parentheses. $* * * p<0.01, * * \mathrm{p}<0.05,{ }^{*} \mathrm{p}<0.1$.

The results show that the coefficients of the institutional quality are significant and positive in all regressions, which indicates that the improvement of institutional quality has a positive effect on the social identity of migrant workers. Moreover, among all factors, the coefficients of institutional quality are the biggest in all models, which suggests that the institutional quality is the most important factors affecting the integration of Chinese migrant workers.

Table 3 reports the results of how different institutions affect the integration of migrant workers including Hukou, education and social security.

Table 3. Results of different institution

\begin{tabular}{|c|c|c|c|}
\hline & 1 & 2 & 3 \\
\hline Hukou & $\begin{array}{c}0.152 * * * \\
(0.017)\end{array}$ & & \\
\hline Education & & $\begin{array}{c}0.122 * * * * \\
(0.018)\end{array}$ & \\
\hline Social security & & & $\begin{array}{c}0.127 * * * \\
(0.014)\end{array}$ \\
\hline Age & $\begin{array}{c}-0.035^{* *} \\
(0.018)\end{array}$ & $\begin{array}{c}-0.036^{* *} \\
(0.018)\end{array}$ & $\begin{array}{c}-0.039^{* *} \\
(0.018)\end{array}$ \\
\hline Sex & $\begin{array}{c}0.042 * * \\
(0.036)\end{array}$ & $\begin{array}{l}0.054^{*} \\
(0.036)\end{array}$ & $\begin{array}{l}0.057 * * \\
(0.035)\end{array}$ \\
\hline Duration & $\begin{array}{c}0.006 \\
(0.016)\end{array}$ & $\begin{array}{c}0.008 \\
(0.015)\end{array}$ & $\begin{array}{c}0.009 \\
(0.015)\end{array}$ \\
\hline Martial & $\begin{array}{l}0.084 * \\
(0.043)\end{array}$ & $\begin{array}{l}0.076^{*} \\
(0.043)\end{array}$ & $\begin{array}{l}0.079^{*} \\
(0.043)\end{array}$ \\
\hline Income & $\begin{array}{c}0.213 * * \\
(0.0186)\end{array}$ & $\begin{array}{l}0.212 * * \\
(0.0187)\end{array}$ & $\begin{array}{c}-0.213 * * * \\
(0.0187)\end{array}$ \\
\hline Consumption & $\begin{array}{l}0.024^{*} \\
(0.013)\end{array}$ & $\begin{array}{c}0.026^{* *} \\
(0.013)\end{array}$ & $\begin{array}{l}0.023^{*} \\
(0.013)\end{array}$ \\
\hline Leisure & $\begin{array}{l}0.022 * \\
(0.033) \\
\end{array}$ & $\begin{array}{c}0.02 \\
(0.033) \\
\end{array}$ & $\begin{array}{c}0.019 \\
(0.033) \\
\end{array}$ \\
\hline R-squared & 0.573 & 0.564 & 0.567 \\
\hline
\end{tabular}


Note: standard errors are included in the parentheses. $* * * \mathrm{p}<0.01, * * \mathrm{p}<0.05, * \mathrm{p}<0.1 /$

As indicated in this table, the coefficient of the household registration system is the largest, which indicates that the household registration system has the greatest impact on the social integration of migrant workers in various institutional factors.

\section{Conclusion}

This paper comparatively analyzes the situation and impacting factors of social integration of migrant workers from group heterogeneity perspective. The study indicates that the institution quality can promote social integration of migrant workers significantly, income, institution quality and social network are the three most important factors. Household Registration System play the most important role among all these institutions affecting social integration, social insurance ranks second, however, the importance of education is decreasing gradually.

\section{References}

[1]. Myers D, Gao X, Emeka A, The Gradient of Immigrant Age at Arrival Effects on Socioeconomic Outcomes in the US, International Migration Review,2009,43 (1): 205-229.

[2]. Nesdale, D., Mak, A.S., 2000. Immigrant Acculturation Attitudes and Host Country Identification. Journal of Community\& Applied Social Psychology (6):483-495.

[3]. Zagefka H, Brown R. The relationship between acculturation strategies, relative fit and intergroup relations: Immigrant -majority relations in Germany. European Journal of Social Psychology,2002,32 (2): $171-188$. 